\title{
Polyploids and Polyploidy.
}

\section{By C. D. Darlington.}

$\mathrm{W}$ HEN a cell divides to produce two daughter cells having the same genetical properties as itself, the nucleus resolves itself into a definite number of structures, the chromosomes. These, splitting lengthwise, give rise to two identical groups which go to make the daughter nuclei. In this process, known as mitosis, it is evident that, with certain exceptions which need not be gone into here, the chromosomes into which the nucleus resolves itself are always identical in number and form with those which went to constitute it at the preceding division. The chromosome number is said to be constant. It follows that when, in the course of sexual reproduction, two germ-cells unite to form a zygote and their nuclei fuse, the new generation of cells thus established will show at mitosis a new chromosome outfit or ' complement', the sum of those of the two germ-cells. When these are identical the number will be simply doubled, and in these circumstances the number of chromosomes in the germ-cell is said to be 'haploid' and the double number of the zygote is said to be 'diploid' (see Fig. 1). Further, when the

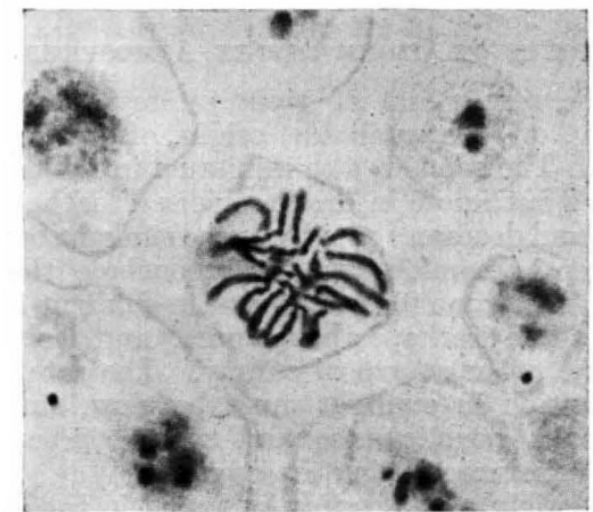

FiG. 1.-The diploid chromosome number (24) in a root tip of Tulipa Clusiana, a form from Tibet.

new zygote comes to produce germ cells, which by their union will reconstitute a diploid individual like itself, the zygote nuclei undergo a process of 'reduction', by which a new cell generation is produced having the haploid number of the gametes.

These three processes provide the mechanism that determines the Mendelian laws of inheritance, and so long as mitosis, fertilisation, and reduction follow a regular course, the Mendelian laws, with all the complications that are implicit in them, are obeyed. But all three processes are liable to error. In the first place, we frequently find body cells, both in plants and animals, the chromosome numbers of which are double those characteristic of the individual and its species. This type of 'doubling' probably follows the failure of two bodies of chromosomes to separate after mitosis, or the simultaneous division of two nuclei which have not been separated by a cell-wall after the preceding cell-division. In plants, owing to their unlimited growth, such 'doubled ' cells sometimes produce germinal tissue, and a new tetraploid race, such as Primula kewensis, with four times the haploid chromosome number, is established. Doubling is so frequent in the formation of a callus in the tomato that we can be certain of obtaining a proportion of tetraploids amongst the adventitious shoots thrown up from the callused wound when the stem is cut off. In the normal course of development, doubling has been found to occur frequently in the embryo-sac nuclei of several plants belonging to the Liliaceæ. Usually the doubling occurs at the opposite end of the embryo sac from the egg-cell, but sometimes the egg-cell itself is affected, and in these cases fertilisation of the diploid female gamete will produce a new triploid individual.

In the second place, abnormalities in fertilisation occur which give rise to a different kind of change. Various stimuli may excite the development of the egg-cell without fertilisation. For example, when an interspecific cross, such as hexaploid Solanum nigrum by tetraploid S. luteum, is attempted, the stimulus of pollination sometimes excites the development of the egg-cell with the reduced (triploid) number, although the pollen-nucleus does not fuse with the egg-nucleus. Similarly, by the stimulus of changes of temperature, an egg-cell in Datura can be induced to develop. In these ways a haploid, or relatively haploid, individual arises having the hereditary material of a germcell and the outward form, on a reduced scale, of a diploid plant. Experimentally it has been found possible to induce the fusion of two male germcells with one female ; in the formation of the endosperm we have virtually the reverse case of the fusion of two female germ-cells with one male. From both these unions a triploid cell generation is produced.

It is in the finely regulated processes of reduction, however, that irregularities most frequently occur, and these irregularities are of great importance in the production of polyploids. In considering reduction, two properties of the chromosomes should be particularly borne in mind. First, the material of which the chromosomes are made up has specific physiological properties, so that, to take a simple example, if one type of chromosome is represented in the organism three times instead of twice, constant physiological changes are produced. To take another example, if one of the chromosomes of a haploid gamete is lacking, the gamete is not usually viable. Secondly, where, as is usually the case, the diploid organism is the result of the union of two similar gametes, the chromosomes contributed by these gametes must correspond in pairs. They must be structurally and functionally homologous. When, therefore, we see pairs of chromosomes formed at reduction, it is a legitimate assumption that these are pairs of homologous

No. 3115, VoL. 124] 
chromosomes and that they pair because they are like. Further, where pairing fails, it is perhaps natural to conclude that this failure is the result of lack of likeness or affinity. Exceptions to this rule we shall return to later. In the main it is true,

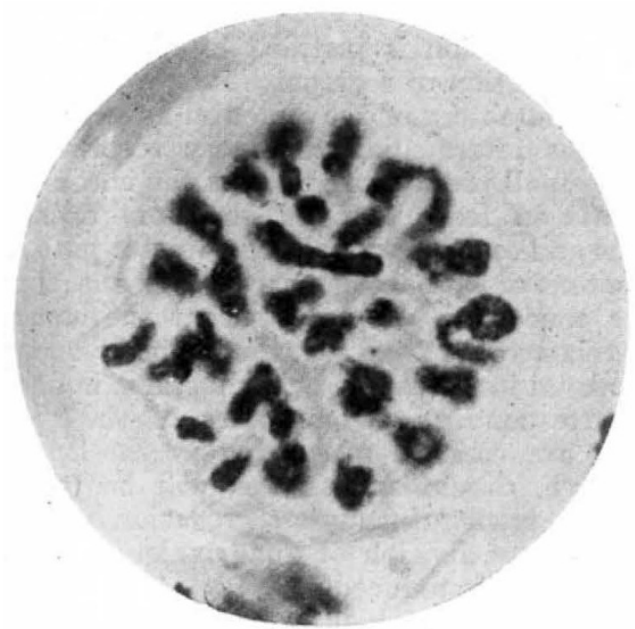

FIG. 2.-Pollen mother-cell division in a pentaploid form of Tulipe Clusiana ( 60 chromosomes) showing associations of various numbers of chromosomes, and some chromosomes unpaired.

and it is as a consequence largely of failure of pairing that irregularities in reduction arise in hybrids.

The simplest form that these irregularities can take is the failure of the two corresponding groups of chromosomes to separate, and the consequent formation of a single nucleus with the diploid number. This nucleus then divides to give two nuclei in which the number is similarly unreduced. Germ-cells have been shown to arise in this way in large numbers of plants, which are either of known hybrid origin (like Raphanus-Brassica) or believed on independent grounds to be hybrids (like species of Hieracium). In Pygora hybrids, where the formation of diploid germ-cells, with complete failure of chromosome-pairing, was first observed, the nuclear phenomenon was shown to be correlated with the fact that the character differences completely fail to segregate. This is one of the discoveries on which the chromosome theory is based.

If the conditions of the origin of polyploids before the fact are almost universal, the conditions after the fact impose important limitations on their maintenance. These limitations are almost all bound up with the processes of sexual reproduction. Where the sexes are differentiated, doubling of the chromosome number may be associated with sterility on one side, as in Pygora hybrids, and consequent failure of the tetraploid to perpetuate itself; or there may be a change in the method of reproduction, as in Artemia where a tetraploid is parthenogenetic, or Empetrum where a tetraploid is hermaphrodite, the diploid that probably corresponds being in each case unisexual.

In a different way the purely mechanical conditions of reduction greatly limit the success of those polyploids which reproduce sexually. The conditions under which the corresponding chromosomes separate regularly when present in pairs are not always equally satisfactory when more than two of each kind are present. For example, in a triploid, where there are three of a sort, the odd chromosomes, whether associated with the pairs or not, separate at random to the two daughter cells. Consequently the germ-cells produced have chromosome numbers varying between the haploid and the diploid number (see Fig. 3). In these new intermediate types the balance of the physiologically differentiated chromosomes is new, untried, and in most cases unsuccessful. Most triploids are therefore sterile so far as sexual reproduction is concerned. Triploidy is none the less common both in natural species and in cultivated plants, but a study of its incidence shows that in these established triploid forms sexual reproduction has lost its value. Triploids occur, for example, in plants with various kinds of natural vegetative propagation in Hyacinthus, Tulipa, Iris, Rubus (Fig. 4), Canna, and Tradescantia; with reproduction by grafting in Prunus; with apogamy in Hieracium; with partial parthenocarpy in the cultivated apples; and probably with both apogamy and parthenocarpy in cultivated Citrus. To such forms as these it is clear that ordinary seed fertility, so far from being an advantage, may actually become a hindrance.

The case of tetraploids is more complicated, both in regard to chromosome behaviour and to the fertility that largely depends on it. For the sake of simplicity, let us take two opposite types. From a haploid Datura, itself the result of the development of an unfertilised egg, diploid offspring have

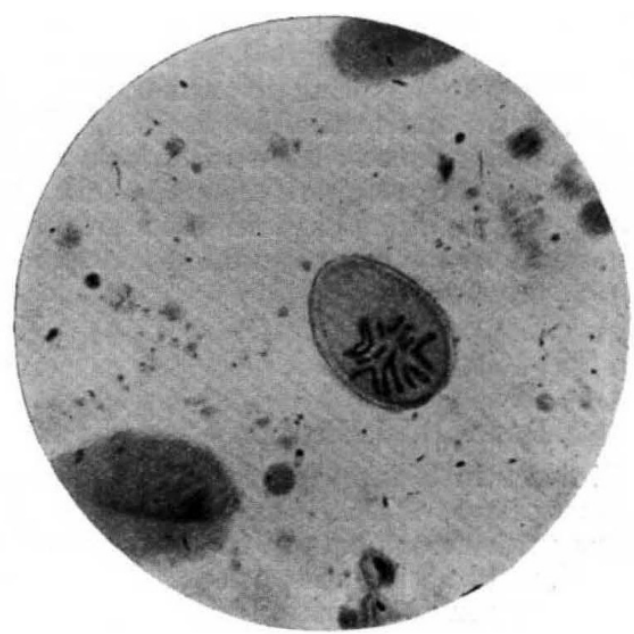

FIG. 3.-A pollen grain of a triploid hyacinth (somatic number, 24) showing 13 chromosomes; numbers between 8 and 16 have been found. Note the three chromosome types.

arisen by failure of reduction, and from these again tetraploids. In such tetraploids there are four identical representatives of each chromosome type, which usually associate to form quadrivalent bodies at reduction. These quadrivalents are, of course, capable of dividing into two and two, but seem to

No. 3115, VoL. 124] 
be subject to irregularity in division, for gametes arise (as in tetraploid Prunus cerasus and Tradescantia virginiana) both with more and less than the normal gametic number, and the fertility of such tetraploids is consequently reduced.

We may mention parenthetically that, just as the functional gametes must contain two out of the four chromosomes of each type chosen at random, so the breeding results in tetraploid Datura and Primula sinensis have always agreed with the assumption that two Mendelian factors, chosen at random from four, pass to each gamete. This agreement, verifying prediction, is a substantial corroboration of the chromosome theory of heredity.

On the other hand, we have tetraploids arising as a result of doubling in hybrids. The result depends on what we may loosely call the 'degree of hybridity.' Primula kewensis is not an extreme example, and will serve to show the peculiarities

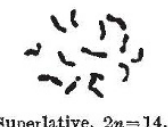

Superlative, $2 n=14$

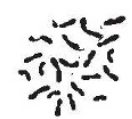

Mahdi, $2 n=21$.

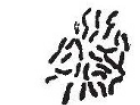

Veitchberry, $2 n=28$.

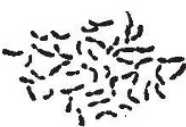

Loganberry, $2 n=42$
Laxtonberry $2 n=49$.

FIG. 4.-Diploid and polyploid species and varieties of Rubus. Al the even multiples are fertile, although the tetraploid and hexaploid are known to be interspecific hybrids. The odd multiples are both sterile in a high degree.

associated with various degrees of hybridity. It is the result of a cross between Primula floribunda and by $P$. verticillata, which gave in the first instance a sterile intermediate diploid hybrid, as might have been expected. This diploid produced a giant fertile shoot the offspring of which were fairly constant, giant, and fertile like itself. This shoot was tetraploid and must have arisen as a result of the formation of tetraploid somatic cells, as in Solanum. In the diploid hybrid, the corresponding chromosomes of the two parental species paired at reduction ; its sterility must therefore be assumed to be the result of failure of the new genetic combinations brought together in the gametes. In the tetraploid, however, pairs are usually formed as in the diploid, but the general absence of segregation means that identical mates must pair and pass to opposite gametes.

This conclusion is justified by the fact that a small proportion of segregates is produced bearing certain characters of the parental species, that is, no longer intermediate in every respect. In a similar small proportion of cases the chromosomes associate in fours, derived therefore from both species and capable of yielding gametes pure in the characters of one species carried by the chromosomes concerned. It follows that the constancy and relative fertility of the hybrid tetraploid depends on its degree of hybridity, for, in so far as the corresponding chromosomes of the opposite species are capable of pairing in the tetraploid derivative, dissimilar gametes will be produced, both as a result of genetic segregation and abnormality in the division of quadrivalents. In both cases fertility is reduced.

These remarks, which are based on theoretical considerations, are in agreement with all the available experimental evidence. Fertile diploids such as Enothera Lamarckiana, Datura Stramonium, Primula sinensis, Solanum Lycopersicum, and Campanula persicifolia give less fertile tetraploids. Sterile diploids such as Primula kewensis, RaphanusBrassica, and relatively diploid Nicotiana and Solanum hybrids give more fertile tetraploids.

It is therefore possible for a tetraploid arising from a hybrid diploid to have the mechanical properties of a fertile diploid. But it combines with these certain genetical peculiarities. Not only is it a hybrid, with such physiological advantages as hybridity may confer, but occasionally the corresponding chromosomes of the opposite diploid parents may pair. The tetraploid will then show the segregation of a hybrid, and may give rise to offspring with any workable combination of the characters of its two parents.

(To be continued.)

\section{Obituary.}

Prof. R. J. Harvey-Gibson.

THE death in Glasgow on June 3, at sixty-nine 1 years of age, of Dr. Robert J. Harvey-Gibson, emeritus professor of botany in the University of Liverpool, will be widely regretted. Prof. HarveyGibson was a son of the manse and was educated in the Universities of Aberdeen, Edinburgh, and Strasbourg. He first held appointments as demonstrator in zoology and later in physiology in the University of Edinburgh. His association with the University of Liverpool goes back to the year 1883, in which his first appointment was that of demonstrator in biology in the old School of Medicine, which was later amalgamated with University College as the foundation of the present University.
At the amalgamation, Harvey-Gibson was promoted to a lectureship in botany, which became in 1887 a subject in the new Faculty of Science. The institution and growth of a Department of Botany under his direction soon followed, and in 1889 a new laboratory was erected to meet the needs of the Department. The first assistant in the Department was Dr. A. J. Ewart, and when in 1894 Mr. Holbrook Gaskell made possible the foundation of a chair of botany, Harvey-Gibson became its first holder and worked arduously for the advancement of the Department and the acquisition of modern laboratories. At that time Dr. J. C. Willis was a student in the Department. In $1900 \mathrm{Mr}$. Hartley offered to build a new laboratory, and in 1902 the present Department was erected to the plans of 